\section{Avaliação da atenção à gestação de alto risco em quatro metrópoles brasileiras}

\author{
Assessment of care for high-risk pregnancy in \\ four Brazilian metropolises
}

\section{Evaluación de la atención a la gestación de alto riesgo en cuatro metrópolis brasileñas}

\author{
Juliana Azevedo Fernandes 1 \\ Sonia Isoyama Venâncio 2 \\ Dário Frederico Pasche 3 \\ Fernanda Luz Gonzaga da Silva 4 \\ Nathan Aratani 4 \\ Oswaldo Yoshimi Tanaka 4 \\ Patricia Rodrigues Sanine 4 \\ Gastão Wagner de Sousa Campos 1
}

doi: 10.1590/0102-311X00120519

\section{Resumo}

Avaliou-se a atenção à gestação de alto risco, incluindo o acesso, o funcionamento e a utilização dos serviços de saúde, desde a atenção primária à saúde (APS) até a atenção especializada. Trata-se de pesquisa avaliativa ancorada em modelo analítico que utiliza a triangulação de diferentes fontes de informações e análise comparativa da atenção à gestação de alto risco em quatro metrópoles brasileiras. As categorias de análise selecionadas foram: acesso a consultas e exames, vínculo entre os componentes das redes de atenção à saúde e entre usuário e profissional e o cuidado oferecido. Caracterizaram-se os contextos de atenção à gestação de alto risco por meio de indicadores dos sistemas de informação em saúde e de estudo descritivo qualitativo produzido por informantes-chave, além de questionário aplicado a 1.886 gestantes dos serviços especializados, encaminhadas pela APS. A triangulação dos dados obtidos por fontes secundárias, contextualização das redes de atenção e inquérito junto às gestantes de alto risco permitiram abarcar a complexidade dos arranjos or ganizacionais da rede de atenção à saúde entre os municípios. Foram identificadas diferenças significativas no acesso, vínculo e cuidado na atenção à gestação de alto risco nas quatro metrópoles estudadas. Campinas (São Paulo, Brasil) destacou-se como o município que alcançou melhor desempenho nos indicadores relacionados a vínculo e acesso das gestantes à APS. São Paulo e Porto Alegre (Rio Grande do Sul) apresentaram desempenho regular no conjunto dos indicadores, ao passo que Fortaleza (Ceará) demonstrou necessidade de melhoria nas três categorias analisadas.

Cuidado Pré-Natal; Gravidez de Alto Risco; Assistência Integral à Saúde;

Pesquisa sobre Serviços de Saúde; Atenção Primária à Saúde

\section{Correspondência}

J. A. Fernandes

Departamento de Saúde Coletiva, Universidade Estadual de Campinas.

Rua Tessalia Vieira Camargo 126, Campinas, SP 13083-887, Brasil.

fernandes.juliana@gmail.com

1 Departamento de Saúde Coletiva, Universidade Estadual de Campinas, Campinas, Brasil.

2 Instituto de Saúde, Secretaria de Estado da Saúde de São Paulo, São Paulo, Brasil.

3 Universidade Federal do Rio Grande do Sul, Porto Alegre, Brasil.

${ }_{4}^{4}$ Faculdade de Saúde Pública, Universidade de São Paulo, São Paulo, Brasil. 


\section{Introdução}

Passadas mais de três décadas do início da implementação do sistema de saúde nacional, com caráter público e acesso universal, o Sistema Único de Saúde (SUS) acumula importantes avanços, como o aumento no acesso da população a consultas médicas e a ampliação do número de procedimentos executados e de serviços de saúde, especialmente de atenção primária à saúde (APS) 1,2,3.

Como estratégia para a organização desse sistema, o Brasil aposta, desde o final de 2010, na estruturação das Redes de Atenção à Saúde (RAS) 4, constituídas por cinco componentes: APS, Atenção Especializada, Sistemas de Apoio, Sistemas Logísticos e Governança. As RAS buscam maior integração territorial, além de ampliar o acesso de qualidade aos serviços de saúde por meio de mecanismos de coordenação do cuidado e economia de escala 5,6,7.

Como sistema de base territorial, os serviços de APS são compreendidos como porta de entrada prioritária e principais ordenadores dessa rede 5. Apesar das diferentes configurações organizacionais, têm no modelo de Estratégia Saúde da Família (ESF) a principal estratégia para melhorar as condições de saúde da população 3,8 .

Entre as cinco redes temáticas priorizadas no SUS, a Rede Cegonha foi a primeira a ser instituída para fomentar a implementação de um novo modelo de atenção à saúde da mulher e da criança, garantindo acolhimento, ampliação do acesso, melhoria da qualidade do pré-natal, vinculação da gestante aos serviços e qualificação das ações voltadas ao parto, ao nascimento e ao recém-nascido, com vistas à redução da mortalidade materna e neonatal ${ }^{9}$.

No Brasil, ainda que avanços tenham que ser conquistados, a ampliação do acesso por meio da APS foi capaz de produzir, como resultado, a redução das taxas de internação por condições sensíveis à APS e de mortalidade infantil 3,10,11,12.

Por outro lado, essa ampliação do acesso desencadeou novos desafios ao sistema de saúde, como em relação à capacidade de governança entre os distintos serviços, resultando na dificuldade para estabelecer fluxos integrados e contínuos de comunicação, além do aumento da demanda por atendimentos e procedimentos em outros níveis de complexidade 5 .

Assim, embora a gestação de alto risco não represente uma patologia específica, o fato de sua magnitude expressa em cerca de $15 \%$ do total de gestações serem de alto risco (aproximadamente 470 mil gestações ao ano no Brasil) 13, associado à chance de ocorrência de desfechos desfavoráveis à mulher, ao feto e ao recém-nascido, justifica a necessidade de realização de estudos sobre essa linha de cuidado. Dessa forma, o presente artigo tem por objetivo avaliar a atenção à gestação de alto risco, incluindo o acesso, o funcionamento e a utilização dos serviços de saúde, desde a APS até a atenção especializada.

\section{Métodos}

Pesquisa avaliativa, de corte transversal, ancorada em um modelo analítico que utiliza a triangulação de diferentes fontes de informações 14,15,16 e análise comparativa da atenção à gestação de alto risco em quatro cidades brasileiras com mais de um milhão de habitantes. Trata-se de um recorte da pesquisa Inquérito sobre o Acesso a partir da Atenção Básica, o Funcionamento e a Utilização da Atenção Especializada para Quatro Condições Traçadoras em Quatro Grandes Cidades Brasileiras - AcesSUS, que analisou o acesso aos serviços a partir da APS, além do funcionamento e da utilização da atenção especializada para três agravos (hipertensão arterial grave, câncer de mama e transtorno mental grave), além da gestação de alto risco.

Os municípios de Campinas (São Paulo), Fortaleza (Ceará), Porto Alegre (Rio Grande do Sul) e São Paulo foram selecionados pela magnitude populacional e, consequentemente, pela importância que representam ao sistema de saúde, além da heterogeneidade que apresentam na composição e organização dos serviços e suas RAS, possibilitando verificar diferenças na organização da atenção à gestação de alto risco, tanto na APS quanto na atenção especializada. Outro fator que favoreceu a eleição dos municípios foi a viabilidade institucional de organizar uma pesquisa multicêntrica com universidades que contemplassem as diversidades regionais do país. 
A escolha do método se deu por apresentar maior adequação para atender às necessidades postas em avaliações de sistemas complexos, superando os desenhos focalizados, restritos e com escassez de instrumentos de coleta de dados 17,18.

Partindo do pressuposto de que a relação que a gestante estabelece com o sistema de saúde depende do seu acesso ao acompanhamento de pré-natal e de como ocorre o percurso entre os serviços da RAS para a garantia de uma atenção integral e qualificada 19, optou-se pela definição de três categorias de análise: acesso da gestante a consultas, exames e medicamentos; vínculo da gestante com os serviços de pré-natal; e o cuidado oferecido à gestante.

Acesso, vínculo e cuidado são conceitos estratégicos para se compreender e avaliar sistemas, serviços e práticas de saúde, mas nem sempre são abordados e explorados de forma adequada. Barbosa \& Bosi 20, analisando a utilização do conceito de vínculo no campo da Saúde Coletiva, apontam que essa categoria tem sido, mormente, associada às de acesso, cuidado e mesmo outras, conformando amálgamas semânticos que, na maioria das vezes, não estabelecem diferenciações entre os termos, criando-se um campo nebuloso.

De outra parte, a natureza polissêmica desses conceitos exige, a partir de uma clara definição semântica, que se construam indicadores para expressar e traduzir sentidos conceituais prévios, uma vez que não haveria, por antecipação, indicadores disponíveis que pudessem, de imediato, expressar as opções conceituais originárias, sobretudo de investigações que se utilizassem de modelos de análise mais complexos, como neste estudo.

Isso posto, tomou-se o acesso como a "oportunidade de utilização dos serviços em circunstâncias que permitam o uso apropriado dos mesmos" 21 (p. 264), uso este que se circunscreve à disponibilidade de oferta de ações e serviços de saúde, mas condicionado a fatores sociais, econômicos e culturais. Logo, a acessibilidade decorreria tanto de ações no âmbito do sistema e serviços de saúde, como de ações intersetoriais e transversais 21 .

O vínculo foi compreendido como produção imaterial decorrente da relação entre sujeitos, vetor para a construção de pertença e reconhecimento do outro como legítimo demandante e cuidador, sustentando contratos terapêuticos e oferta de atenção ajustada às necessidades singulares dos sujeitos. Vínculo, nessa acepção, guarda dois sentidos inter-relacionados: qualidade das relações/encontros que permite intercâmbios de afetos e produção de contratos terapêuticos (afetividade e relação terapêutica); e referência para a atenção em determinados serviços e equipes (vinculação e continuidade). Vinculação, como um dos sentidos de vínculo, foi o que se buscou verificar no âmbito desta pesquisa.

Por fim, cuidado foi tomado como expressão do propósito ético de contração de responsabilidade pelo outro, resultante das relações intercessoras, combinadas pelo uso e manejo de boas práticas de atenção à saúde.

O estudo foi realizado em fases sequenciais, iniciado com a utilização de dados secundários, extraídos dos sistemas oficiais de informação em saúde do SUS. Para tanto, foram consultados: Cadastro Nacional de Estabelecimentos de Saúde (CNES), Sistema de Informação sobre Nascidos Vivos (SINASC), Sistema de Informações Ambulatoriais (SIA) ou equivalente, Sistema de Informações Hospitalares (SIH), dados demográficos do Instituto Brasileiro de Geografia e Estatística (IBGE) e Fundação Sistema Estadual de Análise de Dados (Fundação Seade), entre os meses de outubro de 2015 e fevereiro de 2016.

Com base nas informações contidas nesses sistemas, fez-se o esforço de caracterizar indicadores de acesso (porcentagem de pré-natal adequado e mais do que adequado e razão de ultrassom obstétrico morfológico/nascidos vivos), de vínculo (proporção de pré-natal com sete ou mais consultas) e de cuidado (proporção de parto cesáreo).

$\mathrm{Na}$ fase seguinte, foi coletada a primeira parte dos dados primários, por meio de consulta a informantes-chave de cada município, entre os quais, coordenadores de áreas técnicas das Secretarias Municipais de Saúde e de centrais de regulação e coordenadores de serviços de saúde. Esses dados tiveram caráter qualitativo e foram estruturados por um estudo descritivo, padronizado, contendo características do contexto de funcionamento da rede de atenção à gestação de alto risco em cada um dos sistemas de saúde locais.

Essa coleta foi realizada entre os meses de março e junho de 2017 e buscou a descrição de singularidades e diferenças entre os quatro municípios em relação aos serviços de APS (cobertura populacional e gestão administrativa), disponibilidade de rede de apoio aos serviços de APS (número de 
equipes e cobertura de Núcleos de Apoio à Saúde da Família - NASF), implantação da Rede Cegonha (número de maternidades habilitadas Tipo II), além da articulação entre APS e atenção especializada (critérios para encaminhamento, responsáveis pela regulação e mecanismo de marcação de consultas na atenção especializada).

As informações provenientes dessas duas fases foram articuladas, possibilitando a incorporação do contexto na análise da caracterização da atenção às gestantes.

A terceira fase do estudo contou com a coleta de novos dados primários, que ocorreu por meio da aplicação de questionário junto a gestantes residentes nos municípios e encaminhadas pela APS para acompanhamento na atenção especializada devido à gestação de alto risco (critérios de inclusão). Assim, foram entrevistadas todas as gestantes nessas condições, que estivessem aguardando consulta na atenção especializada no período estipulado para a coleta e que aceitassem ser entrevistadas antes da consulta.

O cálculo amostral levou em consideração o tempo de permanência em campo, que foi calculado conforme a estimativa do número de consultas mensais às gestantes e o número de serviços de atenção especializada de cada município. Definiu-se que a permanência dos entrevistadores seria igual em todos os serviços, garantindo que a distribuição pelos serviços fosse semelhante na amostra e na população de estudo. $O$ tamanho da amostra prevista foi calculado considerando $p=0,50 ; z=1,96$; $\mathrm{d}=0,05$ para todos os municípios, exceto São Paulo, cujo cálculo amostral pode ser expresso por $\mathrm{p}=$ 0,$50 ; z=1,96 ; d=0,04$.

A amostra final para as entrevistas considerou, portanto, o tempo de coleta em relação ao número de serviços visitados, de gestantes a serem abordadas e o de gestantes atendidas semanalmente, totalizando 1.886 gestantes entrevistadas.

A partir desses critérios, as entrevistas com as gestantes foram realizadas, entre os meses de março e setembro de 2016, em 28 serviços de acompanhamento à gestação de alto risco dos respectivos municípios. Foram pesquisados os serviços de especialidades de nível ambulatorial e hospitalar nos municípios que estavam formalmente integrados ao sistema de regulação municipal, acessados pelos serviços de APS e que aceitaram participar.

Utilizou-se um questionário padronizado, previamente testado em dois dos municípios, composto de 49 questões fechadas, das quais quatro abordaram as características sociodemográficas das gestantes e treze eram relativas a informações sobre a atenção à gestação de alto risco no município.

Para a caracterização das gestantes, considerando a influência do contexto socioeconômico 22,23,24, foram consideradas as seguintes variáveis: idade, cor da pele, escolaridade e se possuía plano de saúde e/ou convênio médico. Para a caracterização da atenção à gestação de alto risco, foram utilizadas 13 variáveis, distribuídas entre as categorias acesso, vínculo e cuidado.

As variáveis de acesso utilizadas foram: início precoce do pré-natal na APS; tempo oportuno de espera entre encaminhamento da APS e consulta na atenção especializada; agendamento de consulta de retorno na atenção especializada após consulta; retirada dos medicamentos na APS; realização de todos os exames no SUS; pagamento por uma ou mais consultas para o pré-natal; pagamento por um ou mais exames relacionados ao pré-natal; e conhecimento da maternidade em que ocorreria o parto.

Para a categoria vínculo, utilizaram-se as seguintes variáveis: manutenção do pré-natal na APS após encaminhamento para atenção especializada e conhecimento do agente comunitário de saúde (ACS) da sua área de residência.

Para o cuidado, selecionaram-se variáveis relacionadas à responsabilização institucional, à flexibilidade na interação entre os serviços e à expectativa do usuário na obtenção adequada de informações para a interação: possibilidade de negociar com o médico a forma de parto, se recebeu visita domiciliar durante o pré-natal e se conhece o motivo do encaminhamento para o pré-natal especializado.

As análises do inquérito foram realizadas no pacote estatístico SPSS v.20.0 (https://www.ibm. com/), utilizando pesos amostrais para os serviços de cada cidade e o teste de independência entre linhas e colunas, considerando o peso de junção para amostras complexas a um nível de significância menor que 0,05 .

Para fins de comparação dos resultados observados nos diferentes municípios, decidiu-se pela junção dos bancos de dados referentes aos quatro municípios do estudo. No banco final, foram incluídos novos pesos que consideraram a participação relativa da amostra de cada município na amostra total. Os pesos foram definidos pela relação entre o número de pacientes atendidos no ano 
e e o de pacientes atendidos no período de coleta de dados, o que equivaleria ao inverso da fração de amostragem.

Prevendo a não participação de algum serviço, em municípios em que um dos serviços existentes não participou da pesquisa, os pesos foram corrigidos em função dessa não cobertura, pelo inverso da taxa de cobertura.

O cálculo amostral e o questionário detalhado podem ser acessados no endereço eletrônico https://www.fcm.unicamp.br/acessus/metodologia/inquerito-amostragem.

Todos os resultados foram analisados por meio da comparação entre os municípios em relação às categorias acesso, vínculo e cuidado, buscando identificar a influência dos diferentes contextos sobre os achados.

Este estudo foi aprovado pelo Comitê de Ética em Pesquisa da Universidade Estadual de Campinas (Unicamp) sob o parecer no 1.777.800/2016. Antes da aplicação de cada questionário, foi realizada a leitura do termo de consentimento livre e esclarecido (TCLE), assinado por todas as mulheres que concordaram em participar do estudo, conforme Resolução no 466/2012 do Conselho Nacional de Saúde.

\section{Resultados}

Os dados da Tabela 1 evidenciam limitações relacionadas a todas as categorias nos quatro municípios avaliados. Chamou atenção a categoria cuidado, caracterizado pela alta proporção de partos cesáreos em todos os municípios.

No entanto, apesar dessas limitações, Campinas destaca-se como o município que alcançou melhor desempenho nos indicadores relacionados a vínculo e acesso das gestantes à APS. São Paulo e Porto Alegre apresentaram desempenho regular no conjunto dos indicadores, ao passo que Fortaleza demonstrou necessidade de melhoria nas três categorias analisadas.

Em relação ao contexto de funcionamento da rede de atenção à gestação de alto risco nesses municípios, constataram-se baixas coberturas de serviços de APS, especialmente em Fortaleza e em Campinas. Ainda sobre esses dois municípios, destaca-se a gestão da APS realizada por administração direta, ao passo que, em Porto Alegre, é realizada por uma Fundação Pública de Direito Privado (FPDP) e, em São Paulo, a gestão da APS é terceirizada para Organizações Sociais - OS (Quadro 1).

No Quadro 1, é possível observar, ainda, que São Paulo, Campinas e Fortaleza foram municípios com menor número de serviços de APS cobertos pelo NASF. Porto Alegre se destaca como sendo o

\section{Tabela 1}

Distribuição dos indicadores de caracterização de contexto da atenção à gestação e respectivas fontes de dados, conforme categoria de análise. AcesSUS, 2016.

\begin{tabular}{|c|c|c|c|c|c|}
\hline Indicador (ano da informação) & Campinas & Fortaleza & Porto Alegre & São Paulo & Fontes \\
\hline \multicolumn{6}{|l|}{ Acesso } \\
\hline $\begin{array}{l}\text { Porcentagem de pré-natal adequado * e mais do que } \\
\text { adequado (2016) }\end{array}$ & 77,68 & 55,56 & 70,96 & 77,46 & SINASC \\
\hline $\begin{array}{l}\text { Razão de unidades de saúde obstétrico morfológico/ } \\
\text { Nascidos vivos (2015) }\end{array}$ & 1,62 & 0,67 & 0,88 & 1,12 & SIA e SINASC \\
\hline \multicolumn{6}{|l|}{ Vínculo } \\
\hline Proporção de pré-natal com 7 ou mais consultas (2015) & 79,55 & 56,81 & 74,44 & 76,04 & SINASC \\
\hline \multicolumn{6}{|l|}{ Cuidado } \\
\hline Proporção de parto cesáreo (2016) & 68,79 & 66,70 & 54,52 & 55,71 & AIH-SIH e SINASC \\
\hline
\end{tabular}

AIH: Autoriazação de Internação Hospitalar; SIA-SUS: Sistema de Informação Ambulatorial; SIH: Sistema de Informação Hospitalar; SINASC: Sistema de Informações sobre Nascidos Vivos; SUS: Sistema Único de Saúde.

* Início do pré-natal no primeiro trimestre e um mínimo de seis ou mais consultas de pré-natal. 


\section{Quadro 1}

Descrição do contexto de funcionamento da rede de atenção à gravidez de alto risco. Campinas (São Paulo), Fortaleza (Ceará), Porto Alegre (Rio Grande do Sul) e São Paulo, Brasil. AcesSUS, 2016.

\begin{tabular}{|c|c|c|c|c|c|}
\hline \multicolumn{2}{|l|}{ Variáveis } & Campinas & Fortaleza & Porto Alegre & São Paulo \\
\hline \multirow[t]{2}{*}{ APS } & Cobertura populacional * & $63,30 \%$ & $62,68 \%$ & $67,02 \%$ & $59,53 \%$ \\
\hline & Administração da APS ** & Direta & Direta & $\begin{array}{l}\text { Predominantemente } \\
\text { por Fundação Pública } \\
\text { de Direito Privado }\end{array}$ & $\begin{array}{c}\text { Predominantemente } \\
\text { por OS }\end{array}$ \\
\hline Rede de apoio & $\begin{array}{l}\text { Número de equipes } \\
\text { (cobertura) NASF * }\end{array}$ & $3(13,00 \%)$ & $26(28,00 \%)$ & $8(57,00 \%)$ & $111(14,00 \%)$ \\
\hline Rede Cegonha & $\begin{array}{l}\text { Número de maternidades } \\
\text { habilitadas (Tipo II)** }\end{array}$ & 1 & 1 & 5 & 2 \\
\hline \multirow{3}{*}{$\begin{array}{l}\text { Articulação } \\
\text { APS e atenção } \\
\text { especializada }\end{array}$} & $\begin{array}{c}\text { Critérios de } \\
\text { encaminhamento ** }\end{array}$ & Utilização de protocolo & $\begin{array}{l}\text { Não utilização de } \\
\text { protocolo }\end{array}$ & $\begin{array}{l}\text { Utilização de } \\
\text { protocolo }\end{array}$ & Utilização de protocolo \\
\hline & $\begin{array}{l}\text { Responsável pela } \\
\text { regulação ** }\end{array}$ & $100 \%$ via UBS & $100 \%$ via UBS & $\begin{array}{c}\text { 50\% via regulação } \\
\text { ordinária } \\
\text { 50\% via UBS }\end{array}$ & $100 \%$ via UBS \\
\hline & $\begin{array}{c}\text { Mecanismo de marcação } \\
\text { de consultas atenção } \\
\text { especializada ** }\end{array}$ & $\begin{array}{c}\text { Sistema informatizado } \\
\text { (um municipal e } \\
\text { um estadual) }\end{array}$ & $\begin{array}{c}\text { Telefone ou } \\
\text { agendamento direto } \\
\text { com o hospital de } \\
\text { referência }\end{array}$ & $\begin{array}{c}\text { Sistema informatizado } \\
\text { municipal }\end{array}$ & $\begin{array}{c}\text { Sistema informatizado } \\
\text { (um municipal e } \\
\text { um estadual) }\end{array}$ \\
\hline
\end{tabular}

APS: atenção primária à saúde; NASF: Núcleos de Apoio à Saúde da Família; OS: Organizações Sociais; UBS: unidades básicas de saúde. Fonte: Ministério da Saúde. e-Gestor. https://egestorab.saude.gov.br/paginas/acessoPublico/relatorios/relHistoricoCoberturaAB.xhtmlaginas/ acessoPublico/relatorios/relHistoricoCoberturaAB.xhtml (acessado em Abr/2019).

* Informação e gestão da atenção básica;

** Informantes-chaves.

município que apresenta maior cobertura de NASF (57\%) e o maior número de maternidades habilitadas Tipo II (5). Fortaleza é o único município que não utiliza protocolo de encaminhamento e não possui sistema informatizado para marcação de consulta na atenção especializada.

Com base nas 1.886 gestantes entrevistadas nos serviços especializados em gestação de alto risco (Campinas n = 405; Fortaleza $n=401$; Porto Alegre $n=391$; São Paulo $n=689$ ), pode-se constatar que a maioria de mulheres tinha idade entre 19 e 35 anos (66,97\%), com 9 a 12 anos de estudo (65,53\%), autodeclarou-se parda (à exceção de Porto Alegre) e não possuía plano de saúde e/ou convênio médico $(89,66 \%)$ (Tabela 2).

A Tabela 3 permitiu observar importantes diferenças entre os municípios. No tocante às variáveis relacionadas ao acesso, diferenças estatisticamente significativas foram identificadas em todas as variáveis, exceto início precoce do pré-natal e tempo oportuno de espera entre encaminhamento pela APS e consulta na atenção especializada. Em relação às demais variáveis indicativas de acesso, Campinas mostrou melhor desempenho, e Fortaleza, a situação menos favorável, especialmente na retirada de medicamentos na unidade de saúde e no pagamento por exames. É importante destacar o alto percentual de gestantes que relataram ter pagado por consultas e por exames (Tabela 3). Chamou atenção em São Paulo que menos da metade das gestantes soube relatar a maternidade onde teriam o parto.

Diferenças significativas também foram identificadas em relação às duas variáveis indicativas de vínculo das gestantes com os serviços. Em São Paulo, foi identificado o maior percentual de gestantes 


\section{Tabela 2}

Frequências das variáveis de caracterização das gestantes de alto risco, segundo o município, Campinas (São Paulo), Fortaleza (Ceará), Porto Alegre (Rio Grande do Sul) e São Paulo, Brasil. AcesSUS, 2016

\begin{tabular}{|c|c|c|c|c|c|c|}
\hline Variável & $\begin{array}{c}\text { Campinas } \\
\text { [n = 405] } \\
n(\%)\end{array}$ & $\begin{array}{c}\text { Fortaleza } \\
\text { [n = 401] } \\
n(\%)\end{array}$ & $\begin{array}{c}\text { Porto Alegre } \\
\begin{array}{c}\text { [n = 391] } \\
n(\%)\end{array}\end{array}$ & $\begin{array}{c}\text { São Paulo } \\
\text { [n = 689] } \\
n(\%)\end{array}$ & $\begin{array}{c}\text { Total } \\
{[n=1.886]}\end{array}$ & Valor de $p$ * \\
\hline Idade (em anos) & & & & & & $<0,001$ \\
\hline Até 18 & $17(4,2)$ & $66(13,0)$ & $16(4,1)$ & $78(11,2)$ & 177 & \\
\hline $19-35$ & $298(73,8)$ & $264(68,6)$ & $280(71,8)$ & $421(65,0)$ & 1.263 & \\
\hline 36 ou mais & $89(22,0)$ & $69(18,4)$ & $94(24,1)$ & $190(26,8)$ & 442 & \\
\hline Cor de pele & & & & & & $<0,001$ \\
\hline Branca & $149(37,1)$ & $72(15,5)$ & $223(57,6)$ & $256(36,8)$ & 700 & \\
\hline Preta & $74(18,4)$ & $57(13,6)$ & $110(28,4)$ & $121(16,9)$ & 362 & \\
\hline Parda & $172(42,8)$ & $248(66,8)$ & $51(13,2)$ & $298(44,5)$ & 769 & \\
\hline Amarela & $5(1,2)$ & $15(3,9)$ & $3(0,8)$ & $9(1,5)$ & 32 & \\
\hline Indígena & $2(0,5)$ & $1(0,2)$ & $0(0,0)$ & $1(0,3)$ & 4 & \\
\hline Escolaridade (em anos) & & & & & & 0,009 \\
\hline 4 ou menos & $17(4,2)$ & $18(4,6)$ & $13(3,3)$ & $18(2,0)$ & 66 & \\
\hline $5-8$ & $70(17,3)$ & $89(23,0)$ & $84(21,5)$ & $140(20,8)$ & 383 & \\
\hline $9-12$ & $281(69,6)$ & $241(59,4)$ & $259(66,2)$ & $455(68,1)$ & 1.236 & \\
\hline 13 ou mais & $36(8,9)$ & $53(13,0)$ & $35(9,0)$ & $73(9,1)$ & 197 & \\
\hline \multicolumn{7}{|l|}{$\begin{array}{l}\text { Possui plano de saúde ou } \\
\text { convênio médico }\end{array}$} \\
\hline Não & $364(89,9)$ & $360(90,2)$ & $348(89,0)$ & $619(89,3)$ & 1.691 & 0,877 \\
\hline
\end{tabular}

Fonte: Inquérito AcesSUS, 2016.

* Referente ao teste de independência entre linhas e colunas, considerando o peso de junção para amostras complexas.

que mantiveram pré-natal na APS após encaminhamento para atenção especializada. Campinas foi o município em que as gestantes menos relataram conhecer o agente comunitário de saúde, e Fortaleza, o local em que mais se conhece esse profissional (Tabela 3).

Em relação ao cuidado, diferenças foram encontradas nas três variáveis selecionadas. Em Campinas, houve menor frequência de gestantes que receberam visitas domiciliares. São Paulo se destacou pelo maior percentual de visitas domiciliares, mas foi o local onde o menor número de gestantes pôde negociar a forma de parto. Em Porto Alegre, identificou-se situação favorável em relação à possibilidade de negociar o tipo de parto e receber visitas domiciliares (Tabela 3).

A triangulação dos dados obtidos por fontes secundárias, contextualização das redes de atenção e inquérito junto às gestantes de alto risco permitiu abarcar a complexidade dos arranjos organizacionais da RAS e seus possíveis reflexos nas categorias de análise.

Em Campinas, mais da metade da população estava coberta por serviços de APS. Além disso, a rede de atenção à gestação de alto risco é caracterizada pela APS sob gestão direta e pelo estabelecimento de mecanismos de regulação dos encaminhamentos da APS para a atenção especializada, possibilitando um bom acesso das gestantes de alto risco a consultas na APS e na atenção especializada, exames e medicamentos. Por outro lado, as gestantes relataram pouco vínculo com a APS após o encaminhamento à atenção especializada.

Fortaleza também se caracteriza pela APS sob gestão direta. Os indicadores obtidos pelos dados secundários e as informações provenientes das gestantes mostraram consistência e apontam fragilidades no tocante ao acesso das gestantes a consultas, exames e medicamentos. Por outro lado, foi o município em que houve maior possibilidade de a gestante negociar o tipo de parto. 


\section{Tabela 3}

Frequências das variáveis de caracterização da atenção às gestantes de alto risco, segundo o município, Campinas (São Paulo), Fortaleza (Ceará), Porto Alegre (Rio Grande do Sul) e São Paulo, Brasil. AcesSUS, 2016.

\begin{tabular}{|c|c|c|c|c|c|c|}
\hline Variável & $\begin{array}{l}\text { Campinas } \\
\text { [n = 405] } \\
n(\%)\end{array}$ & $\begin{array}{c}\text { Fortaleza } \\
\text { [n = 401] } \\
n(\%)\end{array}$ & $\begin{array}{l}\text { Porto Alegre } \\
\qquad[n=391] \\
n(\%)\end{array}$ & $\begin{array}{l}\text { São Paulo } \\
\text { [n = 689] } \\
n(\%)\end{array}$ & $\begin{array}{c}\text { Total } \\
{[n=1.886]}\end{array}$ & Valor de $p$ * \\
\hline \multicolumn{7}{|l|}{ Acesso } \\
\hline \multicolumn{7}{|c|}{ Início precoce do pré-natal na APS } \\
\hline 1ㅇ trimestre & $345(85,6)$ & $316(79,5)$ & $334(86,1)$ & $560(81,6)$ & 1.555 & 0,123 \\
\hline \multicolumn{7}{|c|}{$\begin{array}{l}\text { Tempo oportuno de espera entre } \\
\text { encaminhamento da APS e consulta na } \\
\text { atenção especializada ** }\end{array}$} \\
\hline Sim & $247(63,7)$ & $251(64,7)$ & $296(77,7)$ & $463(67,0)$ & 1.257 & 0,154 \\
\hline \multicolumn{7}{|c|}{$\begin{array}{l}\text { Saiu da primeira consulta do pré-natal de } \\
\text { alto risco com retorno agendado }\end{array}$} \\
\hline Sim & $391(97,0)$ & $395(98,0)$ & $377(97,4)$ & $641(93,8)$ & 1.804 & 0,001 \\
\hline \multicolumn{7}{|c|}{ Retirada de todos os medicamentos na } \\
\hline \multicolumn{7}{|c|}{ APS } \\
\hline Sim & $246(69,7)$ & $97(24,8)$ & $167(51,9)$ & $376(61,4)$ & 886 & $<0,001$ \\
\hline \multicolumn{7}{|c|}{ Realização de todos os exames pelo SUS } \\
\hline Sim & $289(94,8)$ & $220(53,9)$ & $306(94,2)$ & $422(82,1)$ & 1.237 & $<0,001$ \\
\hline \multicolumn{7}{|c|}{$\begin{array}{l}\text { Pagou por uma ou mais consultas para o } \\
\text { pré-natal }\end{array}$} \\
\hline Não & $370(91,4)$ & $323(80,6)$ & $323(82,6)$ & $610(90,0)$ & 1.626 & $<0,001$ \\
\hline \multicolumn{7}{|c|}{ Pagou por um ou mais exames } \\
\hline Não & $234(57,8)$ & $72(14,8)$ & $135(34,5)$ & $289(41,9)$ & 730 & $<0,001$ \\
\hline \multicolumn{7}{|c|}{ Sabe em qual maternidade será o parto } \\
\hline Sim & $273(68,8)$ & $236(62,4)$ & $273(70,5)$ & $345(45,5)$ & 1.127 & $<0,001$ \\
\hline \multicolumn{7}{|l|}{ Vínculo } \\
\hline $\begin{array}{l}\text { Manutenção do p } \\
\text { após encaminhan } \\
\text { especializada }\end{array}$ & & & & & & \\
\hline Sim & $163(40,6)$ & $111(24,9)$ & $167(43,0)$ & $501(77,5)$ & 942 & $<0,001$ \\
\hline \multicolumn{7}{|c|}{ Conhece agente comunitário de saúde } \\
\hline Sim & $155(38,3)$ & $269(64,2)$ & $206(53,2)$ & $398(58,6)$ & 1.028 & $<0,001$ \\
\hline \multicolumn{7}{|l|}{ Cuidado } \\
\hline \multicolumn{7}{|c|}{$\begin{array}{l}\text { Negociação da forma de parto com o } \\
\text { médico }\end{array}$} \\
\hline Sim & $44(35,5)$ & $80(43,1)$ & $48(35,3)$ & $49(26,0)$ & 221 & 0,002 \\
\hline \multicolumn{7}{|c|}{$\begin{array}{l}\text { Recebeu visita domiciliar durante o pré- } \\
\text { natal }\end{array}$} \\
\hline Sim & $56(13,8)$ & $169(41,3)$ & $125(32,1)$ & $299(44,2)$ & 649 & $<0,001$ \\
\hline \multicolumn{7}{|c|}{ Motivo do encaminhamento na atenção } \\
\hline Desconhecem & $4(1,0)$ & $3(0,6)$ & $1(0,3)$ & $12(1,6)$ & 20 & $<0,001$ \\
\hline
\end{tabular}

APS: atenção primária à saúde; SUS: Sistema Único de Saúde.

Fonte: Inquérito AcesSUS, 2016.

* Referente ao teste de independência entre linhas e colunas, considerando o peso de junção para amostras complexas;

** Tempo de espera máximo entre encaminhamento da APS e primeira consulta na atenção especializada até 14 dias para gestações no terceiro trimestre e até 28 dias nas demais gestações. 
Porto Alegre possui a maior cobertura populacional de APS, predominantemente sob gestão de uma FPDP, maior disponibilidade de apoio matricial por equipes NASF e protocolos e sistema informatizado para regulação dos encaminhamentos da APS para a atenção especializada. Na análise comparativa com os demais municípios, seus dados apontam uma situação razoável em todos os indicadores das três categorias de análise.

Por fim, São Paulo diferencia-se dos demais municípios pela gestão da APS, predominantemente por OS, e utiliza protocolos e sistema informatizado para encaminhamentos da gestação de alto risco da APS para a atenção especializada. As principais dificuldades identificadas foram o baixo percentual de gestantes que conhecem a maternidade onde terão o parto, que pode ser um indicativo de problemas de acesso à atenção hospitalar, e, em relação ao cuidado, a pouca possibilidade de negociação do tipo de parto.

\section{Discussão}

Este estudo possibilitou a avaliação da atenção a gestantes de alto risco em quatro municípios brasileiros situados entre os 14 com maior população (São Paulo, $n=12.106 .920$; Fortaleza, $n=2.609 .716$; Porto Alegre, $\mathrm{n}=1.481 .019$; Campinas, $\mathrm{n}=1.173 .370$ ), que apresentam índices de desenvolvimento humano maior do que a média nacional, além de possuírem uma rede de equipamentos públicos de saúde vinculados ao SUS, que lhes dão status de centros de referência em saúde em suas respectivas regiões (IBGE. https://cidades.ibge.gov.br, acessado em 21/Abr/2019).

Os resultados permitiram evidenciar a inter-relação entre as categorias de análise que apontaram para a existência de discrepâncias significativas entre as distintas cidades na garantia de acesso, vínculo e cuidado à gestação de alto risco.

O tamanho do SUS e a sua heterogeneidade nos territórios fazem com que os processos avaliativos de seu desempenho sejam um desafio permanente aos pesquisadores, que buscam por meio de seus resultados subsidiar tomada de decisão que oriente o desenvolvimento e o aprimoramento de políticas, programas e estratégias de saúde 25 .

Os métodos adotados permitiram a identificação de diferentes contextos e arranjos organizacionais nessa linha de cuidado e, nessa perspectiva, a aplicação do questionário junto às usuárias já inseridas nos serviços especializados permitiu avaliar o trajeto terapêutico das gestantes de alto risco encaminhadas da APS para a atenção especializada dentro da rede de saúde do SUS em cada um dos municípios.

Estudos apontam diferenças nas configurações do SUS, tanto regionais (norte-sul; capitais-interior), quanto em relação às redes temáticas de atenção 26,27 (Ministério da Saúde. e-Gestor. https:// egestorab.saude.gov.br/paginas/acessoPublico/relatorios/relHistoricoCoberturaAB.xhtml, acessado em Abr/2019). Nos municípios avaliados, essa diferença pode ser evidenciada pela porcentagem de cobertura da APS, na qual se destacam com maior cobertura Porto Alegre e São Paulo, apesar de todos estarem abaixo dos $70 \%$.

Essa baixa cobertura de serviços de APS em municípios de grande porte, especialmente aqueles organizados segundo a ESF, pode ser justificada por restrições financeiras, que impedem o aumento de gastos com pessoal no setor público. Esse é um dos motivos apontados por Costa 28 como indutor da transferência da gestão administrativa desses serviços para OS, o que poderia explicar os arranjos administrativos identificados em São Paulo e Porto Alegre.

Apesar de alguns autores apontarem a associação entre administração direta e melhor desempenho na APS 29, o presente estudo demonstrou que, em relação à gestação de alto risco, os fatores relacionados ao contexto dos municípios interferem muito mais no bom funcionamento dos serviços do que o modelo de gestão administrativa.

Dentre as atribuições da Rede Cegonha, destaca-se a necessidade da captação precoce das gestantes, a partir do acolhimento e avaliação de risco e vulnerabilidades, e a garantia do acesso a um número mínimo de consultas de pré-natal na APS, além do encaminhamento para a atenção especializada em tempo oportuno, quando necessário. No entanto, o avanço na dimensão de acesso à atenção ao pré-natal, por meio da captação precoce das gestantes e ampliação do número de consultas, ainda apresenta desafios sobre seus aspectos qualitativos 30,31 . 
Considerando o risco potencial para o quadro de saúde das gestantes de alto risco, o tempo entre o encaminhamento pela APS e o atendimento da gestante pela atenção especializada apresenta-se como um importante indicador de qualidade ${ }^{32}$. Ainda que os municípios avaliados não tenham apresentado diferenças estatisticamente significantes, chama atenção o número de gestantes que informaram não ter acessado em tempo oportuno os serviços de atenção especializada.

Nesse sentido, o uso de instrumentos como protocolos clínicos de encaminhamento e sistemas de regulação são apontados como estratégias positivas que reduzem esse tempo entre o encaminhamento e a primeira consulta 32 . Vale ressaltar, entre os achados do presente estudo, o protagonismo da APS no que diz respeito aos encaminhamentos das gestantes para os serviços de atenção especializada em todos os municípios estudados, com exceção de Porto Alegre. Ainda que não seja possível identificar claramente a APS como a coordenadora do cuidado no contexto das redes de atenção, tais resultados apontam para um cenário positivo de possível transição dos modelos de regulação, com efetivo reconhecimento das atribuições e do papel da APS nas RAS 33.

O acesso a exames, a consultas e a medicamentos pode ser apontado como outro indicador de qualidade na atenção, pois favorece, inclusive, o início precoce do acompanhamento pré-natal pela APS 22. Conforme apontado em outros estudos, os resultados apresentaram discrepâncias entre os distintos municípios, especialmente se considerado o número de gestantes (acompanhadas no SUS) que afirmaram ter pagado por consultas e/ou exames 34,35 .

Em relação a exames complementares e medicamentos via SUS, Campinas destacou-se como o município que mais disponibilizou acesso, ao passo que Fortaleza foi o município com maiores limitações nessa categoria.

Segundo Guimarães et al. 36, essa restrição na oferta de exames e de medicamentos pode ser justificada pela elevada inadequação estrutural dos serviços de APS, que, diante da limitação de acesso imposto aos serviços públicos de saúde para oferta de atendimento adequado à gestação, fortalece a iniquidade de acesso relativa aos aspectos sociodemográficos dessas gestantes, além de refletir baixa interação entre os distintos serviços de saúde 37.

A influência desses aspectos que privilegiam gestantes brancas, com escolaridade de nível médio e com capacidade de pagamento por atendimento na rede privada, é divulgada pela literatura 22,31,38 e pôde ser mais bem evidenciada nas diferenças existentes entre as gestantes de Fortaleza e Porto Alegre.

As restrições apresentadas pela porcentagem de pré-natal inadequado e razão de exames de ultrassom obstétrico morfológico por nascidos vivos, em Fortaleza, podem ser mais bem compreendidas pelo contexto de funcionamento da rede, que evidencia baixa cobertura de APS, além de inexistência de um protocolo de encaminhamento e de sistema de regulação de vagas para atenção especializada.

Agrava esse contexto o fato de Fortaleza apresentar os indicadores socioeconômicos mais baixos, entre os quatro municípios estudados (Índice de Desenvolvimento Humano = 0,754 e Produto Interno Bruto per capita R\$ 22.092,58 - https://cidades.ibge.gov.br, acessado em 21/Abr/2019), e um perfil de gestantes mais jovens, com predomínio da cor da pele preta e parda e que mais informou ter utilizado a rede privada para a realização de consultas e/ou exames.

Um outro indicador de acesso, mas também relacionado à utilização de protocolos, é o fato de a quase totalidade das gestantes entrevistadas sair da primeira consulta de pré-natal de alto risco com o retorno já agendado. Embora, em uma primeira leitura, esse procedimento possa parecer positivo, cabe refletir sobre sua necessidade, uma vez que uma porção considerável desses encaminhamentos poderia ser evitada e resolvida na APS 32,39.

Cabe destacar, ainda, que esse encaminhamento para a atenção especializada, muitas vezes desnecessário, pode gerar impactos no acompanhamento dessas gestantes junto aos serviços de APS, simbolizando uma inadequada fixação na atenção especializada e/ou a descontinuidade do acompanhamento em um dos níveis de atenção, conforme verificado pelas baixas porcentagens de gestantes que afirmaram realizar acompanhamento compartilhado entre APS e atenção especializada.

Assim, pode-se considerar o acompanhamento compartilhado como resultado de um encaminhamento mais criterioso ${ }^{39}$, mas também influenciado pela construção do vínculo 40 e, nessa perspectiva, São Paulo demonstrou ser o município que melhor manteve vínculo com as gestantes de alto risco.

O ACS é apontado como importante ator em todo esse processo de seguimento do usuário pela APS, devendo manter o vínculo e monitorar o acompanhamento, mesmo após o encaminhamento 
do usuário para os diversos serviços de saúde ${ }^{41}$. Desse modo, conhecer o ACS é um dos fatores que favorecem a atenção compartilhada da gestação de alto risco entre a APS e atenção especializada 22.

No entanto, chama atenção o fato de Fortaleza ser o município que concentrou maior número de gestantes que relataram conhecer os ACS, ao mesmo tempo em que apresentou menor número de gestantes que mantiveram acompanhamento pré-natal na APS. Essa situação, associada ao baixo número de consultas realizadas nesse município, exalta a discussão em relação à construção do vínculo, que não deve ser direcionada apenas aos ACS, mas a toda equipe do serviço 40.

As quatro cidades estudadas apresentaram cobertura de APS em valores próximos, porém o município de Campinas, ainda que estruturado como APS expandida, apresentou baixo protagonismo do ACS em comparação aos demais municípios.

Mais importante do que a presença do ACS no território, a estratégia de visita domiciliar representa um qualificador para a manutenção do cuidado nos serviços de saúde, favorecendo o cuidado compartilhado entre a APS e a atenção especializada 22,42.

Cabe destacar que o desempenho de São Paulo, apesar de ser o município que apresentou maior concentração de gestantes acompanhadas nos dois tipos de serviços e que receberam visita domiciliar, foi um dos piores em relação aos demais indicadores da categoria de análise cuidado. Por outro lado, Fortaleza e Porto Alegre se destacaram quanto à oportunidade de negociar, junto ao médico, a forma de parto e de conhecer o motivo do seu encaminhamento para a atenção especializada.

Nessas duas metrópoles, tais resultados podem ser interpretados como maior autonomia e corresponsabilização das gestantes pelo seu próprio cuidado. Chama, ainda, atenção a categoria de análise "cuidado", que apresentou uma distribuição mais heterogênea entre os municípios, comprovando o quanto as outras categorias exercem influência sobre seu resultado.

As distinções na distribuição desses indicadores pelos quatro municípios são influenciadas por diferentes fatores, tais como a comunicação interprofissional e a qualificação profissional, instituídas pelo NASF, que apresentam elevada capacidade de fortalecimento da APS 41,43,44,45.

No presente estudo, essa organização da rede de atenção à gestação de alto risco pode ser observada na integração dos indicadores que apontam para a cobertura de NASF, a utilização de sistemas informatizados de encaminhamento e a regulação desdas gestantes, resultando no acompanhamento compartilhado entre APS e atenção especializada. Ainda que seja um valor aquém do desejado, esses resultados fortalecem os estudos que apontam as regiões Sul e Sudeste como as que apresentam maior faixa de integração entre serviços 43,44,45.

Convém pontuar algumas limitações reconhecidas no presente estudo, como em relação à complexidade da análise das categorias acesso, vínculo e cuidado diante do alcance restrito do instrumento de coleta de dados, que não avançou para aspectos qualitativos sobre as informações obtidas. Também houve limitações resultantes da articulação de um grupo de pesquisa para trabalhar conjuntamente por quatro anos integrado à perspectiva multicêntrica, como por exemplo, a impossibilidade de contemplar municípios de grande porte das regiões Norte e Centro-oeste do país.

Entretanto, apesar das limitações postas, buscou-se favorecer a inserção de duas cidades de importância reconhecida na Região Sudeste do país, representada por Campinas, que apresenta especificidades na composição da APS e administração 100\% municipal, e o Município de São Paulo, maior cidade do país, com relevante história de implementação das políticas públicas de saúde e de serviços de APS, atualmente com grande peso das OS na administração das unidades.

Assim, pode-se afirmar que tais esforços permitiram identificar contextos que influenciam a organização das redes de atenção à gestação de alto risco e que ainda carecem de ser melhorados, com a implementação de dispositivos de regionalização, formalização e institucionalização de equipes de referência e de apoio matricial ao nível primário e das práticas de comunicação, informação e regulação compartilhada. Os resultados ainda chamam atenção para a necessidade de melhor diagnóstico das vulnerabilidades territoriais para compor a (re)organização da atenção à gestação de alto risco, construção de redes e utilização de protocolos bem definidos quanto aos fluxos locais. 


\section{Colaboradores}

J. A. Fernandes, S. I. Venâncio, D. F. Pasche, F. L. G. Silva, N. Aratani, O. Y. Tanaka, P. R. Sanine e G. W. S. Campos realizaram contribuições substanciais para a concepção do trabalho, interpretação de dados, elaboração do trabalho e aprovação final da versão a ser publicada e acordaram em prestar contas de todos os aspectos da pesquisa.

\section{Informações adicionais}

ORCID: Juliana Azevedo Fernandes (0000-00016057-5058); Sonia Isoyama Venâncio (0000-00017147-3292); Dário Frederico Pasche (0000-00017161-8607); Fernanda Luz Gonzaga da Silva (0000-0002-2576-458X); Nathan Aratani (00000002-4602-7319); Oswaldo Yoshimi Tanaka (00000002-5653-0794); Patricia Rodrigues Sanine (00000002-7668-0327); Gastão Wagner de Sousa Campos (0000-0001-5195-0215).

\section{Agradecimentos}

Os autores agradecem a colaboração dos serviços de atenção à gestação, em especial, às usuárias que responderam ao inquérito, além do Grupo de Pesquisa AcesSUS, responsável por todo planejamento e coleta dos dados. A pesquisa foi financiada pelo MCTI/CNPq/MS - SCTIE - DECIT no 41/2013.

\section{Referências}

1. Bahia L. Trinta anos de Sistema Único de Saúde (SUS): uma transição necessária, mas insuficiente. Cad Saúde Pública 2018; 34:e00067218.

2. Paim JS. Sistema Único de Saúde (SUS) aos 30 anos. Ciênc Saúde Colet 2018; 23:1723-8.

3. Pinto Junior EP, Medina MG, Silva MGC. Efeito da Estratégia Saúde da Família nas internações por condições sensíveis à atenção primária em menores de um ano na Bahia, Brasil. Cad Saúde Pública 2018; 34:e00133816.

4. Ministério da Saúde. Portaria no 4.279, de 30 de dezembro de 2010. Estabelece diretrizes para a organização da rede de atenção à saúde no âmbito do Sistema Único de Saúde (SUS). Diário Oficial da União 2010; 31 dez.

5. Santos CM, Barbieri AR, Gonçalves CCM, Tsuha DH. Avaliação da rede de atenção ao portador de hipertensão arterial: estudo de uma região de saúde. Cad Saúde Pública 2017; 33:e00052816.

6. Viana ALd'A, Bousquat A, Melo GA, De Negri Filho A, Medina MG. Regionalização e redes de saúde. Ciênc Saúde Colet 2018; 23:1791-8.

7. Organização Pan-Americana da Saúde. A atenção à saúde coordenada pela APS: construindo as redes de atenção no SUS. Brasília: Organização Pan-Americana da Saúde; 2011.

8. Sanine PR, Zarili TFT, Nunes LO, Dias A, Castanheira ERL. Do preconizado à prática: oito anos de desafios para a saúde da criança em serviços de atenção primária no interior de São Paulo. Cad Saúde Pública 2018; 34:e00094417.

9. Ministério da Saúde. Implantação das Redes de Atenção à Saúde e outras estratégias da SAS. Brasília: Ministério da Saúde; 2014.

10. Venancio SI, Rosa TEC, Sanches MTC, Shigeno EY, Souza JMP. Efetividade da Estratégia Saúde da Família sobre indicadores de saúde da criança no Estado de São Paulo. Rev Bras Saúde Mater Infant 2016; 16:283-93.

11. Viacava F, Oliveira RAD, Carvalho CC, Laguardia J, Bellido JG. SUS: oferta, acesso e utilização de serviços de saúde nos últimos 30 anos. Ciênc Saúde Colet 2018; 23:1751-62.

12. Aquino R, Oliveira NF, Barreto ML. Impact of the Family Health Program on infant mortality in Brazilian Municipalities. Am J Public Health 2009; 99:87-93.

13. Ministério da Saúde. Critérios e parâmetros para o planejamento e programação de ações e serviços de saúde no âmbito do Sistema Único de Saúde. Brasília: Ministério da Saúde; 2015. (Série Parâmetros SUS).

14. Minayo MCS, Assis SG, Souza ER. Avaliação por triangulação de métodos. Rio de Janeiro: Editora Fiocruz; 2005.

15. Tanaka OY, Ribeiro EL, Almeida CAL. Avaliação em saúde: contribuições para incorporação no cotidiano. Rio de Janeiro: Editora Atheneu; 2017

16. Yin RK. Estudo de caso: planejamento e métodos. Porto Alegre: Bookman; 2015. 
17. Hortale VA, Pedroza M, Rosa MLG. Operacionalizando as categorias acesso e descentralização na análise de sistemas de saúde. Cad Saúde Pública 2000; 16:231-9.

18. Carnut L, Narvai PC. Avaliação de desempenho de sistemas de saúde e gerencialismo na gestão pública brasileira. Saúde Soc 2016; 25:290-305.

19. Departamento de Atenção Básica, Secretaria de Atenção à Saúde, Ministério da Saúde. Política Nacional de Atenção Básica. Brasília: Ministério da Saúde; 2017. (Série E. Legislação de Saúde) (Série Pactos pela Saúde 2006, 4).

20. Barbosa MIS, Bosi MLM. Vínculo: um conceito problemático no campo da saúde coletiva. Physis (Rio J.) 2017; 27:1003-22.

21. Sanchez RM, Ciconelli RM. Conceitos de acesso à saúde. Rev Panam Salud Pública 2012; 31:260-8.

22. Sanine PR, Venâncio SI, Silva FLG, Aratani N, Moita MLG, Tanaka OY. Atenção ao pré-natal de gestantes de risco e fatores associados no município de São Paulo. Cad Saúde Pública 2019; 35:e00103118.

23. Sánchez-Gómez A, Cevallos W, Grijalva MJ, Silva-Ayçaguer LC, Tamayo S, Jacobson JO, et al. Factores sociales asociados con la utilización de los servicios de atención prenatal en Ecuador. Rev Panam Salud Pública 2016; 40:341-6.

24. Domingues RMSM, Viellas EF, Dias MAB, Torres JA, Theme-Filha MM, Gama SGN. Adequação da assistência pré-natal segundo as características maternas no Brasil. Rev Panam Salud Pública 2015; 37:140-7

25. Viacava, F, Porto SM, Carvalho CC, Bellido J. Desigualdades regionais e sociais em saúde segundo inquéritos domiciliares (Brasil, 19982013). Ciênc Saúde Colet 2019; 24:2745-60.

26. Conill EM. Ensaio histórico-conceitual sobre a atenção primária à saúde: deságios para a organização de serviços básicos e da Estratégia Saúde da Família em centros urbanos no Brasil. Cad Saúde Pública 2008; 24 Suppl 1:S7-16.

27. Leite JA, Bittencourt CCBLD, Sampaio JF, Leite RA, Cavalcante JC. Efetividade dos princípios do Sistema Único de Saúde na atenção primária à saúde: revisão sistemática. Rev APS 2018; 21:278-90.

28. Costa NR. A Estratégia de Saúde da Família, a atenção primária e o desafio das metrópoles brasileiras. Ciênc Saúde Colet 2016; 21:1389-98.

29. Ramos ALP, De Seta MH. Atenção primária à saúde e Organizações Sociais nas capitais da Região Sudeste do Brasil: 2009 e 2014. Cad Saúde Pública 2019; 35:e00089118.

30. Tomasi E, Fernandes PAA, Fischer T, Siqueira FCV, Silveira DS, Thumé E, et al. Qualidade da atenção pré-natal na rede básica de saúde do Brasil: indicadores e desigualdades sociais. Cad Saúde Pública 2017; 33:e00195815.

31. Mario DN, Rigo L, Boclin KLS, Malvestio LMM, Anziliero D, Horta BL, et al. Qualidade do pré-natal no Brasil: Pesquisa Nacional de Saúde 2013. Ciênc Saúde Colet 2019; 24:1223-32.
32. Silveira MSD, Cazolo LHO, Souza AS, Pícoli RP. Processo regulatório da Estratégia Saúde da Família para a assistência especializada. Saúde Debate 2018; 42:63-72.

33. Almeida PF, Santos AM. Atenção Primária à Saúde: coordenadora do cuidado em redes regionalizadas? Rev Saúde Pública 2016; 50:80.

34. Balsells MMD, Oliveira TMF, Bernardo EBR, Aquino PS, Damasceno AKC, Castro RCMB, et al. Avaliação do processo na assistência prénatal de gestantes com risco habitual. Acta Paul Enferm 2018; 31:247-54.

35. Nunes JT, Gomes KRO, Rodrigues MTP, Mascarenhas MDM. Quality of prenatal care in Brazil: review of published papers from 2005 to 2015. Cad Saúde Colet (Rio J.) 2016; 24:252-61.

36. Guimarães WSG, Parente RCP, Guimarães TLF, Garnelo L. Access to prenatal care and quality of care in the Family Health Strategy: infrastructure, care, and management. Cad Saúde Pública 2018; 34:e00110417.

37. Jongh TE, Gurol-urganci, I, Aleen, E, Zhu NJ, Atun R. Integration of antenatal care services with health programmes in low- and middleincome countries: systematic review. J Glob Health 2016; 6:010403.

38. Fagbamigbe AF, Idemudia ES. Barriers to antenatal care use in Nigeria: evidences from nonusers and implications for maternal health programming. BMC Pregnancy Childbirth 2015; 15:95.

39. Fernandes JA, Campos GWS, Francisco PMB. Perfil das gestantes de alto risco e a cogestão da decisão sobre a via de parto entre médico e gestante. Saúde Debate 2019; 43:406-16.

40. Feuerwerker LCM. Micropolítica e saúde: produção do cuidado, gestão e formação. Porto Alegre: Editora Rede Unida; 2014.

41. Souza FOS, Albuquerque PC, Nascimento CMB, Albuquerque LCL, Lira AC. O papel do Núcleo de Apoio à Saúde da Família na coordenação assistencial da Atenção Básica: limites e possibilidades. Saúde Debate 2017; 41:1075-89.

42. Venkateswaran M, Bogale B, Khader KA, Awwad T, Friberg IK, Ghanem B, et al. Effective coverage of essential antenatal care interventions: a cross-sectional study of public primary healthcare clinics in the West Bank. PLoS One 2019; 14:e0212635.

43. Chaves LA, Jorge AO, Cherchiglia ML, Reis IA, Santos MAC, Santos AF, et al. Integração da atenção básica à rede assistencial: análise de componentes da avaliação externa no PMAQ -AB. Cad Saúde Pública 2018; 34:e00201515.

44. Foy R, Hempel S, Rubenstein L, Suttorp M, Seelig M, Shanman R, et al. Meta-analysis: effect of interactive communication between collaborating primary care physicians and specialists. Ann Intern Med 2010; 152:247-58.

45. Tesser CD, Poli Neto P. Atenção especializada ambulatorial no Sistema Único de Saúde: para superar um vazio. Ciênc Saúde Colet 2017; 22:941-51. 


\section{Abstract}

The study assessed care for high-risk pregnancy, including access, functioning, and use of health services, ranging from primary healthcare (PHC) to specialized care. This was an evaluative study anchored in an analytical model that used triangulation of different information sources and comparative analysis of care for high-risk pregnancy in four Brazilian metropolises. The selected analytical categories were: access to consultations and tests, linkage between components of the healthcare networks and between users and healthcare professionals, and the care provided. Contexts of care for high-risk pregnancy were characterized by health system information indicators and a qualitative descriptive study produced by key informants, in addition to a questionnaire applied to 1,886 pregnant women seen at specialized services, having been referred by PHC. Triangulation of data obtained from secondary sources, contextualization of healthcare networks, and a survey with high-risk pregnant women allowed encompassing the complexity of the organizational arrangements in the healthcare network among the municipalities. Significant differences were identified in access, linkage, and care provided for high-risk pregnancy in the four metropolises. Campinas (São Paulo State, Brazil) was the municipality with the best performance in the indicators related to linkage and access by pregnant women to PHC. São Paulo and Porto Alegre (Rio Grande do Sul State) showed fair performance on the set of indicators as a whole, while Fortaleza (Ceará State) revealed the need for improvement in the three categories analyzed.

Prenatal Care; High-Risk Pregnancy; Comprehensive Health Care; Health Services Research; Primary Health Care

\section{Resumen}

Se evaluó la atención a la gestación de alto riesgo, incluyendo el acceso, funcionamiento y la utilización de los servicios de salud, desde la atención primaria en salud (APS) hasta la atención especializada. Se trata de una investigación evaluativa anclada en un modelo analítico que utiliza la triangulación de diferentes fuentes de información y análisis comparativo de la atención a la gestación de alto riesgo en cuatro metrópolis brasileñas. Las categorías de análisis seleccionadas fueron: acceso a consultas y exámenes, vínculo entre los componentes de las redes de atención a la saludy entre usuario y profesional, así como el cuidado ofrecido. Se caracterizaron los contextos de atención a la gestación de alto riesgo mediante indicadores de los sistemas de información en salud y de estudio descriptivo cualitativo, generado por informantes-clave, además de un cuestionario aplicado a 1.886 gestantes de los servicios especializados, dirigidas por la APS. La triangulación de los datos obtenidos por fuentes secundarias, contextualización de las redes de atención, y encuesta a las gestantes de alto riesgo, permitieron abarcar la complejidad de las disposiciones organizativas de la red de atención en salud entre los municipios. Se identificaron diferencias significativas en el acceso, vínculo y cuidado en la atención a la gestación de alto riesgo en las cuatro metrópolis estudiadas. Campinas (São Paulo, Brasil) se destacó como el municipio que alcanzó un mejor desempeño en los indicadores relacionados con el vínculo y acceso de las gestantes a la APS. São Paulo y Porto Alegre (Rio Grande do Sul) presentaron un desempeño regular en el conjunto de los indicadores, mientras que Fortaleza (Ceará) demostró una necesidad de mejoría en las tres categorías analizadas.

Atención Prenatal; Embarazo de Alto Riesgo; Atención Integral de Salud; Investigación sobre Servicios de Salud; Atención Primária de Salud
Recebido em 26/Jun/2019

Versão final reapresentada em 22/Out/2019 Aprovado em 31/Out/2019 